Meta

Journal des traducteurs

Translators' Journal

\title{
Les dictionnaires polyglottes
}

esquisse historique

\section{Henri Van Hoof}

Volume 39, numéro 1, mars 1994

La traduction et l'interprétation dans la Belgique multilingue

URI : https://id.erudit.org/iderudit/003085ar

DOI : https://doi.org/10.7202/003085ar

Aller au sommaire du numéro

Éditeur(s)

Les Presses de l'Université de Montréal

ISSN

0026-0452 (imprimé)

1492-1421 (numérique)

Découvrir la revue

Citer cet article

Van Hoof, H. (1994). Les dictionnaires polyglottes : esquisse historique. Meta,

39(1), 213-228. https://doi.org/10.7202/003085ar d'utilisation que vous pouvez consulter en ligne.

https://apropos.erudit.org/fr/usagers/politique-dutilisation/ 


\title{
LES DICTIONNAIRES POLYGLOTTES - ESQUISSE HISTORIQUE
}

\author{
HENRI VAN HOOF \\ Institut libre Marie Haps, Bruxelles, Belgique \\ Membre du Comité pour l'histoire de la traduction de la FIT
}

\begin{abstract}
Alors que l'encyclopédie est nécessairement unilingue, puisqu'elle doit servir de source de connaissance à une communauté linguistique déterminée, les dictionnaires, lexiques, glossaires et autres vocabulaires peuvent être unilingues ou multilingues. L'encyclopédie est livre-savoir. Les dictionnaires sont livres-outils et, parmi ceux-ci, les ouvrages bilingues et multilingues représentent un genre bien distinct, répondent à une finalité bien spécifique, celle de la communication. C'est à ce titre, qui en fait des auxiliaires de la traduction, que nous voudrions en évoquer le cheminement historique.

Dans quelle circonstances les dictionnaires bilingues ou multilingues font-ils leur apparition? Quelles sont les conditions qui les font naitre? Existaient-ils dans l'Antiquité? Les civilisations anciennes s'intéressaient-elles aux langues des autres peuples?
\end{abstract}

\section{L'ANTIQUITÉ}

Nous savons que 3000 ans avant J.-C. déjà, l'Égypte est en contact avec le monde extérieur et se livre à un intense commerce international, notamment avec la Mésopotamie, la Nubie, le Sinaï. Nous savons que son activité diplomatique a nécessité la présence de traducteurs et d'interprètes, que, dès l'Ancien Empire, le titre de «chef des interprètes» est une charge héréditaire des princes d'Éléphantine et qu'un hiéroglyphe spécifique représente la fonction. La Genèse (42:24) nous apprend d'ailleurs que lorsque Joseph est rejoint en Égypte par ses frères, ceux-ci «ne savaient pas que Joseph comprenait (ce qu'ils disaient), car il se servait avec eux d'un interprète». Au XIIre siècle avant J.-C., un traité égypto-hittite est traduit en égyptien. Un siècle plus tard, un scribe se targue de savoir le crétois. Tous ces repères permettent de conclure à la connaissance et à la pratique de langues étrangères par une partie des lettrés égyptiens. Pourtant, aucun document lexicographique attestant ces connaissances ou leur enseignement n'est parvenu jusqu'à nous. Chez les Sumériens, il existe, au niveau des scribes tout au moins, un certain bilinguisme, puisque des tablettes découvertes à Nouzi (2500 av. J.-C.) sont écrites en babylonien et en hourrite. Plus tard, lorsque l'akkadien supplante le sumérien, se développe un bilinguisme akkadien-sumérien pour l'étude des textes anciens, qui sont collectionnés, copiés et traduits à la demande des souverains assyriens, eux-même lettrés. Le roi Assurbanipal (668-626) ne confie-t-il pas à l'une des tablettes retrouvées dans sa bibliothèque de Ninive qu'il a «lu les belles tablettes d'argile de Sumer et l'obscure écriture akkadienne, si difficile à maîtriser»? Dès l'instant où les Sémites ont ravi aux Sumériens la primauté politique, leur langue - l'akkadien - s'impose et devient même pour plusieurs siècles la langue des relations diplomatiques et des échanges culturels. Les scribes, qui avaient déjà l'habitude de dresser des listes de mots unilingues, entreprennent, à partir de 1900 av. J.-C., de les doubler d'une autre liste qui donne la traduction akkadienne du signe sumérien correspondant. Ainsi naissent les lexiques bilingues dont divers exemplaires ont été exhumés. Les fouilles d'Ougarit (XIV-XII ${ }^{\mathrm{e}} \mathrm{s}$. av. J.-C.), grande ville cosmopolite à la jonction des empires hittite, mésopotamien et égyptien, ont même mis à jour un lexique quadrilingue

Meta, XXXIX, 1, 1994 
sumérien-akkadien-hourrite-ougaritique. La Perse, elle aussi, connaît un plurilinguisme diplomatique, administratif et commercial. Le livre d'Esther $(8: 9)$ révèle que sous le règne d'Assuérus (ou Xerxès $\mathrm{I}^{\mathrm{er}}, 485-465$ av. J.-C.), l'administration perse envoyait des lettres «aux Juifs, aux satrapes, aux gouverneurs et aux chefs des cent vingt-sept provinces situées de l'Inde à l'Éthiopie, à chaque province selon son écriture, à chaque peuple selon sa langue, et aux Juifs selon leur écriture et leur langue». L'inscription de son père, Darius $\mathrm{I}^{\mathrm{er}}$ (521-486 av. J.-C.), relevée sur le rocher de Behistun est trilingue vieux perseélamite-akkadien. Ces témoignages, pour probants qu'ils soient, ne sont pas confirmés par des documents lexicographiques.

Les anciens Grecs sont à ce point convaincus de la supériorité de leur langue qu'ils laissent aux autres peuples le soin de l'apprendre. En cas de nécessité, ils préfèrent recourir à des interprètes étrangers, comme Xénophon au cours de l'expédition de la mer Noire ou Alexandre le Grand dans sa campagne d'Asie centrale où il recrute des interprètes perses, hyrcaniens, sogdiens et indiens. Thémistocle, qui parlait le perse, constitue une exception. Et il est d'autant plus étonnant de voir un Hesychios ( $\mathrm{V}^{\mathrm{e}} \mathrm{s}$.) consacrer tout un lexique à des termes étrangers. Les Romains, comme les Grecs, n'affichent pas une curiosité très vive pour les langues étrangères. Que le poète Ennius (240-170 av. J.-C.) déclare «avoir trois âmes» parce qu'il sait le latin, le grec et l'osque, ou que le grand Ovide (43 av.-17 apr. J.-C.) ait l'idée d'apprendre le gète pendant son exil sur les bords de la mer Noire, ne change pas grand-chose à cette réalité. Comme les Grecs, les Romains utilisent aussi les services d'interprètes dans leurs relations avec les Égyptiens, les Syriens, les Scythes, les Germains, les Celtes, et bien d'autres peuples encore. Pourtant, même si sa dignité lui interdit de s'exprimer autrement qu'en latin, le Romain cultivé possède le grec dans son bagage intellectuel, car à l'école les deux langues sont enseignées sur un même plan. Cet enseignemenrt fait-il appel à des vocabulaires bilingues? On ne peut que le supposer, car les grammairiens romains n'ont rien légué à la postérité dans ce domaine.

\section{LE MOYEN ÂGE}

$\mathrm{Au}$ Moyen Âge, le savoir est concentré entre les mains de l'Église et la langue de ce savoir est le latin, mais l'évangélisation commande de répandre la bonne nouvelle parmi les masses qui n'entendent pas le latin. Les ecclésiastiques songent alors à dresser des listes d'équivalence latin-langue vulgaire des mots les plus utiles pour la lecture de la Bible et les besoins de la liturgie. C'est l'origine des glossaires bilingues mérovingiens, considérés par d'aucuns comme les ancêtres les plus proches de nos dictionnaires bilingues. Le Glossaire de Reichenau (VII ${ }^{\mathrm{e}} \mathrm{s}$.), 1'un des plus anciens documents pour l'histoire des langues romanes, en est un bel exemple; destiné à faciliter la lecture de la Bible aux populations rhénanes, il propose au mot latin le mot roman correspondant. Même l'enseignement dispensé aux moines requiert de semblables glossaires. Ainsi, en Angleterre, l'abbé d'Eynsham, Aelfric (955-1025), est obligé d'ajouter un lexique latin-saxon à la grammaire latine qu'il écrit pour les religieux de sa congrégation. Par ailleurs, à partir de 1200 , la bourgeoisie montante veut acquérir une connaissance élémentaire du latin, et d'autres vocabulaires bilingues font leur apparition pour répondre à cet enseignement utilitaire, comme aux Pays-Bas le Glossarium Bernense ( $\pm 1290-1310)$ latin-moyen limbourgeois ou le plus ambitieux Vocabularius copiosus (av. 1400) latin-moyen néerlandais (brabançonlimbourgeois) conçu pour étudiants déjà plus avancés.

Le commerce, également, joue un rôle non négligeable dans la création de lexiques bilingues. Ainsi, les Gênois, établis tout autour de la mer Noire depuis la signature d'un traité avec Byzance en 1169, compilent-ils divers vocabulaires latin-coman destinés à faciliter les relations commerciales avec le peuple turc qiptchaq (coman pour les Byzantins, polovtsien pour les Russes). L'un d'entre eux, augmenté du perse par des missionnaires 
franciscains venus pour christianiser les régions pontiques conquises par l'Islam depuis le $\mathrm{XIII}^{\mathrm{e}}$ siècle, devient le lexique trilingue latin-perse-coman connu sous le nom de Codex cumanicus, dont on possède une copie de 1303, mais dont la composition est antérieure à 1295. Lors de l'invasion mongole, de nombreux Comans se réfugient en Égypte, où paraissent bientôt des dictionnaires arabe-turc, tels celui d'Abu al'-Andaluzi al'Garnati (1313) et celui d'Abu Muhammad Abd Allakh am-Turki (début XVe s.). Ainsi donc, malgré la prédominance du latin, on constate que les nécessités du commerce levantin, d'une part, les relations avec le monde musulman, d'autre part, ou encore la stimulation des Croisades (fin $\mathrm{XI}^{\mathrm{e}}$-début XIII ${ }^{\mathrm{e}} \mathrm{s}$.) et, ultérieurement, la volonté de convertir les infidèles, conduisent à l'étude pratique de langues comme le turc et l'arabe, mais aussi le syriaque et l'hébreu.

\section{LA RENAISSANCE : PREMIER ÂGE D'OR}

À la Renaissance, tous les grands philologues, qu'ils soient français, espagnols, flamands ou italiens, connaissent l'hébreu. Les polémiques religieuses nées de la Réforme renforcent l'étude de cette langue, du syriaque, du grec que l'admiration de l'Antiquité remet sur un pied d'égalité avec le latin. Le développement des voyages et des relations internationales favorise le contact avec plus de langues nouvelles et crée un besoin de manuels et d'ouvrages lexicographiques pour les langues étrangères. La Renaissance devient ainsi le premier âge d'or des dictionnaires bilingues et polyglottes.

\section{Les ouvrages basés sur le latin}

Le latin, qui reste le moyen d'expression des lettrés et des savants, sert de langue de départ ou d'arrivée à la plupart des ouvrages. Combiné avec le grec, il donne le Dictionarium graeco-latinum (1539) de l'humainste et lexicographe allemand Peter Rauchfuss, dit Dasypodius (?-1559) et le Dictionarium latino-graecum issu de la collaboration des humanistes allemands Philipp Schwarzerd, dit Mélanchthon (1497-1560), Johachim Camermeister, dit Camerarius (1555-1574) et Johann Lonicer (1499-1569). Le grec et le latin accompagnent aussi d'autres langues dans toute une série d'ouvrages plurilingues, tels le Rerum maxime vulgarium congesta vocabula (1538), petit dictionnaire scolaire latingrec-néerlandais du Hollandais Petrus Curius, le Dictionarium Triglotton (1545) latingrec-néerlandais du Flamand Jan Knaep, dit Servilius, qui connut quinze réimpressions avant la fin du siècle, un Dictionnaire des huit langaiges (grec, latin, flamand, françois, espagnol, italien, anglais, allemand) (1550), le dictionnaire trilingue latin-grec-français Verborum Latinorum cum Graecis Gallicisque coniunctorum Commentarii (1558) de l'humaniste et imprimeur français Guillaume Morel (1505-1564), le Dictionarium tetraglotton (1562) de l'Anversois Cornelis Van Kiel, dit Kiliaan, qui reprend en grande partie Morel pour le latin, le grec et le français en y ajoutant le flamand, le Nomenclator omnium rerum (1567) du médecin et humaniste hollandais Adriaan de Jonghe, dit Junius (1511-1575), sorte de dictionnaire systématique latin avec traduction des entrées en grec, allemand, néerlandais, français, italien, espagnol et parfois anglais. Tous ces travaux doivent beaucoup au Dictionarium (1502) du moine augustin italien Ambrogio Calepino (14351510), qui consacra sa vie à la rédaction de ce lexique d'abord latin-italien, puis augmenté des équivalents en grec, espagnol, allemand, anglais et français, cette dernière version étant assurée en 1565 par André des Morgues (latinisé en Andreas Morgaesius). Dans cet ouvrage, qui bénéficie de dix-huit réimpressions entre 1542 et 1592 et dont le nombre de langues passe de sept à onze, incluant même le polonais et le hongrois, Morel puise pour le grec; à son tour, Kiliaan s'inspire de Morel, et le Pentaglotton qui paraît à Anvers, en 1545 , est encore une adaptation latin-grec-allemand-néerlandais-français de Calepino. 
La combinaison du latin avec le français produit quelques dictionnaires de première

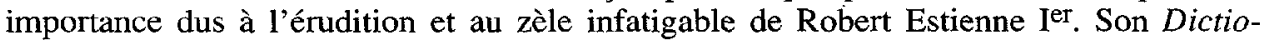
narium Latinogallicum (1538) et son Dictionnaire Françoislatin (1539) fournissent pour la première fois un tableau relativement complet du vocabulaire français. Les impératifs scolaires font abréger le premier, en 1542, sous le titre Dictionariolum puerorum Latinogallicum; le second, en 1544, sous le titre Les mots François, changé plus tard en Petit dictionnaire des mots françois. Un autre Dictionnaire françoislatin paraît en 1564 , sous le nom de Jean Thierry; c'est une version augmentée d'Estienne. Le français intervient aussi dans plusieurs dictionnaires polyglottes qui sortent de presse hors de France. En Flandre, le Dilucidissimus dictionarius imprimé à Anvers en 1534 comprend le latin, le français, le néerlandais, l'espagnol et l'italien; le dictionnaire scolaire de l'humaniste tournaisien Jean Desmarez, dit Paludanus (?-1525), imprimé à Gand sous le titre Dictionariolum rerum maxime vulgarium in communem puerorum usum (1544) est trilingue latin-français-néerlandais; le grand Thesaurus Theutonicae linguae (Schat der Nederduytschen spraken) (1573) d'Andriets Madoets, correcteur de l'imprimerie Plantin à Anvers, donne les mêmes langues mais en partant du néerlandais, et, dans l'esprit de Plantin, il fallait qu'il «se puisse au moins esgaler à ceux des autres nations».

En Angleterre, John Baret (?-1580) inclut le français dans son ouvrage intitulé An Alvearie: or triple Dictionarie in Englishe, Latin and French (1573). Les dictionnaires bilingues sont évidemment les plus nombreux. Déjà, en 1496, le grammairien John Stanbridge avait composé un Latin-English Vocabulary et l'éditeur Richard Pynson avait publié, en 1499, le Promptorium parvulorum sive clericorum, un lexique anglais-latin attribué au dominicain Galfridus Grammaticus. Ce n'est toutefois qu'en 1538 que l'humaniste et diplomate Thomas Elyot (1490-1546), encouragé par le roi Henry VIII, produit le premier véritable dictionnaire latin-anglais, intitulé Bibliotheca Elyotae. Le Thesaurus Linguae Romanae et Britannicae (1545) de l'évêque Thomas Cooper (15171594) est une version augmentée d'Elyot; le Dictionarium Linguae Latinae et Anglicanae (1588) de Thomas Thomas part également du latin, tandis que l'Abecedarium AngloLatinum (1552) de Richard Huloet inverse l'ordre.

L'Allemagne connaît des dictionnaires associant le latin et la langue vulgaire dès la fin du XVe siècle, tel par exemple le Vocabularius qui intitulatur Teuthonista vulgariter dicenda der Duytschender (1477) du chancelier de Clèves, Gert van der Schueren, qui se compose d'un lexique bas rhénan-latin et de son homologue latin-bas rhénan. Le lexicographe Dasypodius, déjà cité, publie en 1535-1537 un Dictionarium Latinogermanicum. Trois autres ouvrages sont l'œuvre d'érudits suisses : le Dictionarium Germanicolatinum (1541) de Johann Fries, dit Frisius (1505-1565), le Dictionariolum puerorum Latinogermanicum (1556) du même Frisius, en collaboration avec Petrus Cholinus, qui se base sur le Latinogallicum d'Estienne, enfin Die Teütsch Spraach. Dictionarium Germanicolatinum novum (1561) du pasteur Josua Maaler (1529-1599), qui est le premier dictionnaire alphabétique à partir de l'allemand.

Aux Pays-Bas, nous avons vu que le néerlandais figure déjà dans de nombreux dictionnaires polyglottes. Associé au seul latin, il n'engendre pas moins d'ouvrages, d'une ampleur et d'une qualité très variables. Le Pappa puerorum (1514), du recteur hollandais Joannes Murmellius (1480-1517), est un petit dictionnaire systématique latinnéerlandais à usage scolaire; le lexique néerlandais-latin Synonymorum Sylva (1537), du moine hollandais Simon Pelegromius, remplit la même fonction. Le premier dictionnaire traductif néerlandais-latin au sens moderne est le Dictionarium Teutonico-latinum (1542) que l'humaniste flamand Antoon Schore, dit Schorus (?-1552), adapte du Latinogermanicum de Dasypodius et qui contient, de ce fait, beaucoup de germanismes. Le Tyrocinium linguae latinae (1552), du Hollandais Pieter van Afferden, dit Apherdanius, est un autre 
dictionnaire scolaire systématique latin-néerlandais. Pour son Dictionariolum Latinogermanicum (1556), à usage scolaire également, le recteur hollandais Ewald Fránssen, dit Gallus, puise le latin dans Calepino. Le plus ambitieux et le plus complet des dictionnaires néerlandais-latins de l'époque sort toutefois des mains de Cornelis Kiliaan, l'éminent lexicographe flamand, correcteur chez Plantin pendant cinquante ans et déjà cité précédemment; il s'agit du Dictionarium Teutonico-latinum (1574) qui, pour la première fois, fait la distinction entre les mots hollandais, flamands, zélandais, frisons, etc. et qui, à partir de la troisième édition (1599), est rebaptisé Etymologicum Teutonicae linguae sive Dictionarium Teutonico-latinum, pour souligner l'importance accordée à l'étymologie.

Pour la combinaison latin-espagnol, le premier dictionnaire date de 1492; c'est le Dictionarium latino-hispanum et hispano-latinum du grammairien Antonio de Nebrija (1444-1532). En Europe du Nord, l'humaniste, traducteur et imprimeur Kristiern Pedersen (1480-1554) est le premier à publier un vocabulaire latin-danois (1510).

\section{Les ouvrages affranchis du latin}

La Renaissance innove par ailleurs en se dotant de dictionnaires complètement affranchis du latin. Le premier du genre parât être un French-English Vocabulary (1480), imprimé par le célèbre Caxton. Pour l'anglais encore, le traducteur et lexicographe William Salesbury (1520-1584) publie, en 1547, A Dictionary in Englishe and Welshe.

La combinaison flamand-français se concrétise dans un premier Vocabulare (1536) de Noël van Berlaimont, instituteur anversois d'origine hennuyère, mais le premier vrai dictionnaire alphabétique partant du flamand est le Naembouck (1546), de l'imprimeur gantois Joos Lambrecht, ouvrage qui prend le nom de Dictionnaire flamen-françois à partir de sa troisième édition de 1582. Un autre instituteur anversois, mais d'origine française, Claude Luython, donne également un Dictionnaire en françois et flameng (1552). Le premier ouvrage alphabétique pour la combinaison français-flamand paraît en 1557 : c'est le Vocabulaire françois-flameng de Gabriel Meurier (1530-1610), basé sur Les mots françois d'Estienne et rebaptisé Dictionnaire François-Flameng en 1574 ; l'auteur, instituteur d'origine hennuyère lui aussi, en donne l'homologue, Dictionnaire flameng-françois, en 1563. De même, Mathias Sasbout, correcteur chez Plantin, publie un Dictionnaire FlamengFrançoys (1576) basé sur le Thesurus de Madoets amputé du latin, et le Dictionnaire François-Flameng (1579) correspondant. Il est imité dix ans plus tard par le Frison E. Mellema, installé à Anvers, qui fait paraître en 1587 un Dictionnaire ou promptuaire Flameng-Françoys et Françoys-Flameng.

\section{LE XVII' SIÈCLE}

À partir du XVII ${ }^{\mathrm{e}}$ siècle, le nombre de dictionnaires consacrés exclusivement aux langues vulgaires ne fait que croître. Curieusement, cependant, il faut noter que c'est seulement au cours de ce siècle que l'intérêt marqué par la Réforme pour des langues comme l'hébreu et le syriaque produit ses effets sous forme lexicographique.

\section{Les langues anciennes}

En Allemagne, l'hébraïsant Johann Buxtorf (1564-1629) publie un Lexicon chaldaicum, thalmudicum et rabbinicum (1607), que son fils, Johann II (1599-1644), complète par un Lexicon chaldaicum et syriacum (1622). En France, le rabbin Philippe d'Aquin (1578-1650) compose un Dictionnaire hébreu-chaldéen-thalmud-rabbinique (1629), bientôt suivi d'un Thesaurus synonymicus hebraico-chaldaico-rabbinicum (1643-45) dû à l'orientaliste Jean Plantavit de La Panse (1576-1651). La plupart de ces ouvrages continuent à user du latin, ainsi d'ailleurs que ceux réservés à la combinaison avec le grec, comme le 
Lexicon ionicum-graeco-latinum et le Lexicon doricum-graeco-latinum publiés en 1603, par le philologue Grec Emile Portus (1550-1612) et le Thesaurus polono-latino-graecus (1621) du jésuite polonais Georges Knapski, dit Knapius (1564-1638).

C'est le cas, plus logiquement encore, des dictionnaires fondés sur le latin, que l'on trouve accouplé aux langues les plus variées. En combinaison avec le flamand paraissent le Dictionarium Teutonico-Latinum (1620-1625), du libraire anversois Martin Binnart, et le Dictionarium Belgico-Latinum (1684), du philologue classique frison Wigardus van Winschoten; le premier, rebaptisé Biglotton sive Dictionarium Teutolatinum novum en 1649, ne connaîtra pas moins de vingt-huit rééditions en moins d'un siècle. En Allemagne, l'orientaliste Job Ludolf (1624-1704) publie un Lexicon aethiopico-latinum (1660), auquel le dominicain Johann Wansleben (1635-1679), son élève pour l'éthiopien, ajoute un Index latinus (1661). L'orientaliste et grammairien polonais Franciszek Meninski (1623-1698) compile un Thesaurus linguarum orientalum (1680) pour l'arabe, le perse et le turc. Pour le turc encore, l'orientaliste français André Du Ryer (1580-1660) compose un Dictionarium turco-latinum, tandis que l'humaniste hongrois Albert de Szencz Molnar (1574-1634) donne à son pays un Dictionarium latino-magyarum.

L'éventail des langues traitées ne cesse de s'élargir. Il est vrai que les travaux sur l'histoire et la description des langues entrepris dès le siècle précédent par un Conrad Gessner (Mithridates, sive de differentis linguarum, 1555) ont montré leur multiplicité. Le Thesaurus polyglottus (1603) de l'Allemand J. Megiser, qui recense 400 langues, et le Trésor de l' histoire des langues de l'univers (1613), du Français Claude Duret, viennent le confirmer. Les lexicographes se trouvent donc devant une tâche immense, et l'intérêt grandissant porté aux langues étrangères pour des raisons didactiques, politiques, commerciales ou autres va se traduire par une multitude de dictionnaires pour les combinaisons linguistiques les plus diverses.

\section{Les dictionnaires affranchis du latin}

Le français, l'anglais demeurent certes les langues de base d'un grand nombre d'ouvrages, mais l'espagnol et le néerlandais en deviennent des rivaux par le jeu des contingences historiques.

La combinaison français-néerlandais s'enrichit d'un Grand dictionnaire FrançoisFlamen (1618) qui est une édition augmentée de Mellema dans lequel on trouve pour la première fois le mot woorden-boec (dictionnaire). Suivent, parmi les plus importants, un Schatkamer der Nederduytsche en Francoysche tale. Le gazophylace de la langue Françoise et Flamende (1654-56) de Caspar van den Ende, instituteur à Rotterdam, et le Dictionnaire nouveau François et Flamand (1686) de François Halma et C. Rouxel. Le français et le néerlandais côtoient l'espagnol dans Den groten dictionaris en de schat van dry talen, Duytsch, Spaensch ende Fransch, qui paraît à Anvers, en 1639, et interviennent aussi dans un Dictionnaire des onze langues (grec, latin, français, hollandais, espagnol, italien, anglais, allemand, gallois, portugais, hébraïque) qui voit le jour à Londres, en 1677. Parmi d'autres ouvrages basés sur le français, il faut citer le Dictionarie of the French and English Tongues (1611) de Randle Cotgrave (?-1634), le Dictionnaire turcfrançais et français-turc de l'orientaliste François Pétis de la Croix (1622-1695) et le dictionnaire multilingue anglais-français-italien-espagnol que le philologue et historien James Howell (1594-1666) publie en 1659-1669, sous le titre Lexicon tetraglotton.

L'anglais se retrouve, associé cette fois au néerlandais, dans plusieurs ouvrages tels A copious English and Netherduytsch dictionarie, composed out of our best English authours (1647) et son homologue Het groot woordenboek: gestelt in 't Nederduytsch ende 't Engelsch (1648) de Henry Hexham, un ancien soldat qui séjourna longtemps dans les Provinces Unies, ainsi que le dictionnaire anglais-néerlandais et néerlandais-anglais 
Nieuw Woordenboek (1691) de Willem Sewel, qui sera l'ouvrage standard pour cette combinaison de langues tout au long du siècle suivant.

Le Vocabulaer (1617) espagnol-néerlandais de Jan Colijn de Thovoyon est le premier d'une série consacrée à l'association de ces deux langues. En 1624, Juan Francisco Rodriguez, instituteur au château d'Anvers, publie un Nieuwen dictionaris néerlandaisespagnol. Il est suivi, en 1650, d'un Dictionaris Duytsch ende Spaensch et, en 1659, Arnoldus de la Porte, chapelain du château d'Anvers, fait paraître son Nuevo dictionario, o thesoro de la lengua Española y Flamenga. Den nieuwen dictionaris oft schadt der Duytse ende Spaensche talen (espagnol-flamand et flamand-espagnol).

Dans un registre plus exotique, le XVII' siècle voit encore la parution d'un Dictionnaire chinois-castillan, que l'on doit au missionnaire Francisco Diaz (1580-1646), et d'un Dictionnaire portugais-annamite, qui est l'œuvre du missionnaire Manoel Ferreira (1631-1699).

\section{LE XVIII SIÈCLE}

Le XVIII' ${ }^{e}$ siècle, faut-il le rappeler, est un siècle encyclopédiste. Dans cet esprit se poursuit l'inventaire des langues parlées dans le monde, inventaire auquel les princes eux-mêmes semblent prendre intérêt. Ne voit-on pas Catherine II de Russie envoyer un questionnaire à tous les gouverneurs de son empire? Les matériaux ainsi collectés sont mis en forme par un ethnographe allemand du nom de P. Sallas, qui en tire un Linguarum totius orbis vocabumaria comparativa (1786-87), sorte de dictionnaire de 285 mots traduits en quelque deux cents langues. Il ne faut donc pas s'étonner si, parallèlement aux encyclopédies, les dictionnaires polyglottes continuent à proliférer, même pour les langues mortes.

\section{Les langues anciennes}

Le grec est encore à I'honneur dans le Greek-English Dictionary du philologue Edmund Barker (1788-1839) et le Grosses kritisches Wörterbuch griechisch-deutsch (1797-98) du philologue Johann Schneider (1750-1822), lequel va servir de modèle à tous les dictionnaires grecs ultérieurs; à Moscou paraît même un dictionnaire slavon-grec-latin en 1704. Le latin, on le trouve encore associé au français dans le Dictionnaire françaislatin (1717) du jésuite François Paumey; au néerlandais dans le Nieuw Woordenboek (1704) de Samuel Hannot et dans le Novum dictionarium Belgico-Latinum publié à Maastricht, en 1717; à 1'allemand dans le Teutsch-lateinisches Wörterbuch (1741) de Johann Frisch, le fondateur de la lexicographie allemande, et dans le Vollständiges lateinisch-deutsches und deutsch-lateinisches Wörterbuch (1783-84) du lexicographe Immanuel Scheller (1735-1803); à l'espagnol, dans le vaste Dictionarium latino-hispanicum de l'érudit Juan de Iriarte (1702-1771); à dix autres langues dans le Dictionarium undecim linguarum (1718), nouvelle adaptation du Calepino par le lexicographe italien Jacopo Facciolati (1682-1769).

\section{Les langues vivantes}

Les dictionnaires les plus nombreux sont évidemment ceux qui ne combinent que des langues vivantes. Pour la combinaison français-néerlandais, le Français Pierre Marin, qui à la fin du siècle précédent avait déjà publié un Dictionnaire portatif Hollandois et François (1696), confirme avec deux ouvrages plus complets, le Nieuw Nederduits en Frans Woordenboek (1701) et son homologue, le Dictionnaire complet Français et Hollandais (1710). Son rival, François Halma, lui oppose son Grand dictionnaire François et Flamend (1708) - qui en sera déjà à sa sixième édition en 1781 , et son Woordenboek der Neder- 
duitsche en Fransche talen (1710) - qui connaîtra déjà sa neuvième édition en 1786. Cela n'empêche pas Jean des Roches, instituteur à Anvers et natif de La Haye, de publier un autre Nieuw Nederduytsch en Fransch woorden-boek (1769). La combinaison françaisespagnol est couverte dès 1705 par le Dictionnaire nouveau des langues française et espagnole que Francisco Sobrino fait paraître à Bruxelles.

Pour l'association français-anglais, l'historien et lexicographe Abel Boyer (16181698), qui se fixa en Angleterre après la révocation de l'édit de Nantes, compose son Dictionnaire royal anglais-français et français-anglais (1727), auquel succède le Nouveau dictionnaire français-anglais et anglais-français (1762) de Chambaud. La combinaison français-allemand s'enrichit d'un Nouveau dictionnaire allemand-français et françaisallemand paru à Strasbourg, en 1774; d'un Neues Wörterbuch der französischen und deutschen Sprachen (1787) publié par Schwan, à Mannheim; d'un Dictionnaire françaisallemand et allemand-français (1784-1785) composé par le lexicographe Jean Thibault de Lavaux (1749-1827). Pour l'italien, Francesco Alberti fait paraître un Nouveau dictionnaire français-italien et italien-français (1771-1772). La fin du siècle voit encore la publication du premier Dictionnaire français-russe (1786).

La langue anglaise, en dehors des ouvrages qui l'associent au français, est encore présente dans le Dizionario italiano-inglese de Giuseppe Baretti (1719-1789) et dans le New English and Dutch Dictionary (1789) que John Holtrop adapte de Sewel en y ajoutant les mots nouveaux tirés du dictionnaire de Samuel Johnson.

Pour l'allemand, le lexicographe Mathias Kramer publie d'abord, en combinaison avec l'italien, Das herrliche grosse Teutsch-Italiänisches Dictionarium (1700-1702), puis, alors qu'il est âgé de 78 ans, en combinaison avec le néerlandais, un Koninklyk NederHoog-Duitsch en Hoog-Neder-Duitsch dictionnaire (1719).

Le néerlandais, pour sa part, bénéficie encore d'un Groot woordenboek (1710) italiennéerlandais et néerlandais-italien de Mozes Giron, et même d'un Tesouro (1714) portugaisnéerlandais et néerlandais-portugais dû à la collaboration d'Abraham Alewijn et Johannes Collé.

$\mathrm{Au}$ rayon des langues non européennes, le Thesaurus de l'orientaliste polonais Meninski paraît dans une nouvelle édition en quatre volumes financée par Marie-Thérèse d'Autriche et rebaptisée Lexicon arabico-persico-turcicum (1780-1802).

\section{LE XIX SIÈCLE}

$\mathrm{Au} \mathrm{XIX}^{\mathrm{e}}$ siècle, sous l'influence de la découverte du sanskrit et de l'importance que lui accordent les linguistes dans la quête d'une langue originelle commune, les travaux lexicographiques prennent une orientation nouvelle et des dciso sanskrits, assyriens, perses et autres fleurissent un peu partout.

\section{Les dictionnaires de sanskrit}

Dès 1819, l'orientaliste anglais Horace Wilson (1786-1860), qui séjourna en Inde de 1811 à 1832, publie un Sanskrit-English Dictionary, auquel font suite un EnglishSanskrit Dictionary (1851) et un Sanskrit-English Dictionary (1872) de Sir MonierWilliams (1819-1899), successeur de Wilson à la chaire de sanskrit à l'Université d'Oxford. Des orientalistes allemands comme Theodor Goldstucker (1821-1872) et Theodor Benfey (1809-1881) choisissent la langue anglaise pour rédiger l'un et l'autre un SanskritEnglish Dictionary, le premier en 1856-1863, le second en 1866. En France, l'orientaliste Émile Burnouf (1821-1907) fait paraître son Dictionnaire classique sanscrit-français (1863-1864). Le perse fait l'objet d'un Short English-Persian Dictionary (1883) de la main de l'orientaliste Edward Palmer (1840-1882). Pour l'hébreu, qui conserve des partisans comme langue-mère, l'Allemagne voit paraître le Hebräisch-chaldäisches Wörterbuch 
(1810) de Wilhelm Genesius (1786-1842) et l'Italie, le Vocabolario ebraico-italiano ed italiano-ebraico (1824) de Francesco Fontanella (1768-1827).

\section{Les dictionnaires de grec}

Le grec, bien que langue morte, demeure bien vivant dans les travaux des lexicographes. On le trouve en combinaison avec les principales langues modernes. Ainsi, pour le français, Joseph Planche (1762-1853) donne, dès 1809, un Dictionnaire grec-français qui est un abrégé du Thesaurus d'Henri Estienne, puis un Dictionnaire français-grec (1824) en collaboration avec Charles Alexandre (1797-1870) et Charles Defauconpret (1797-1865). Un an plus tard, l'helléniste Félix Dehèque (1794-1890) publie un Dictionnaire grec-français moderne (1825). Dans la seconde moitié du siècle paraissent encore un Lexique grec-français (1862) d'Édouard Sommer (1822-1866) et un Dictionnaire grec-français (1894) d'Anatole Bailly (1833-1911). En Angleterre, Henry Lidell (18111898) publie, avec Robert Scott, A Greek-English Lexicon (1843) basé sur le Handwörterbuch der griechischen Sprache paru en 1814-1824 sous le nom du philologue allemand Franz Passow (1786-1833). En Allemagne encore, Valentin Rost (1790-1862) fait paraitre un Griechisch-deutsches Wörterbuch (1820) souvent réédité tout au long du siècle et dont la onzième édition est assurée par Albrecht, qui en donne aussi l'homologue Deutschgriechisches Wörterbuch (1889). En Italie, un Vocabolario greco-italiano (1821) est l'œuvre du philologue Fontanella déjà cité. Enfin, le linguiste slovène Miklosich publie un Lexicon palaeo-slovenico-graeco-latinum (1862-1865).

\section{Les dictionnaires de latin}

Le latin, comme le grec, suscite un nombre appréciable d'ouvrages. En France, le grammairien Jean-François Noël (1755-1841) donne, d'entrée de siècle, un Dictionnaire latin-français (1807) et son homologue français-latin (1808). En 1829, c'est un Nouveau dictionnaire latin-français de Barthélémy de Wailly (1800-1869); en 1844, un Dictionnaire latin-français de Louis Quicherat (1799-1867), suivi de son homologue françaislatin (1858); en 1852, un Dictionnaire latin-français de Napoléon Theil (1808-1878); en 1859, un Lexique français-latin d'Edouard Sommer, déjà cité ; en 1893, un Dictionnaire latin-français du philologue Henri Goelzer (1853-1929). Associé au roman, il produit des ouvrages plus spécialisés comme le Glossaire roman-latin du XVe siècle (1865), du philologue belge Jean Scheler (1819-1890), ou le Lexique latin-roman (1890-1891) du romaniste allemand Gustav Koerting (1845-1913). En Allemagne, Lorenz Diefenbach (1806-1883) publie un Glossarium latino-germanicum (1857), tandis que Karl Georges (1806-1895) procède à une refonte complète du grand dictionnaire de Scheller pour donner un Lateinischdeutsches Wörterbuch (1879-1880), très estimé des latinistes, suivi d'un Deutschlateinisches Handwörterbuch. Le latin se retrouve aussi en compagnie de langues moins banales. Ainsi, l'orientaliste allemand Georg Freytag (1788-1861) compose un Lexicon arabico-latinum (1830-37); son collègue français Auguste Carrière (1838-1902) publie un Ancien glossaire latin-arménien (1886). En France encore, le sinologue Joseph de Guignes fils (1759-1845) est l'auteur d'un Dictionnaire français-latin-chinois et l'abbé Paul Perny (1818-1907), qui fut missionnaire en Chine pendant vingt ans, fait paraître un ouvrage du même titre en 1869. Enfin, on doit à l'écrivain serbe Stefan Karadjitch (17871864) un Serbisch-deutsch-lateinisches Wörterbuch.

\section{Les langues vivantes}

Cette richesse de publications, pourtant impressionnante, n'est rien en regard de la pléthore à laquelle on est confronté dès que l'on se tourne vers les dictionnaires qui combinent les seules langues vivantes. Le français, l'anglais et l'allemand nous serviront 
de guides pour un inventaire forcément plus exemplatif qu'exhaustif. À tout seigneur, tout honneur: le français d'abord.

Dès 1832, il se trouve associé à l'italien dans le Dictionnaire français-italien et italien-français d'Antonio Buttara (1771-1832). Des lexicographes français, allemands et suisses se penchent sur sa combinaison avec l'allemand: Adolphe Régnier (1880-1884) donne un Dictionnaire français-allemand et allemand-français (1841), Wilhelm von Suckau (1798-1869) fait de même en 1846 et Theobald Fix (1802-1874) en 1875. Le plus novateur et le plus complet, cependant, est le remarquable Dictionnaire encyclopédique allemand-français et français-allemand, qui paraît en fascicules entre 1869 et 1880 , à la suite d'un contrat conclu en 1863, par la maison Langenscheidt, avec le romaniste Karl Sachs (1829-1909) et le professeur français Césaire Villatte. La langue anglaise est représentée par un English-French Pocket Dictionary (1872), de John Bellows (1831-1907) et Alexandre Beljame (1842-1906), et par un Dictionnaire anglais-français et françaisanglais (1895) d'Alfred Elwall (1818-1889), professeur anglais naturalisé français. L'helléniste Émile Legrand (1841-1903) se penche sur le grec moderne dans son Nouveau dictionnaire grec moderne-français (1882) et français-grec moderne (1885). Un Dictionnaire arménien-français et français-arménien (1861) est l'œuvre du littérateur Ambroise Calfa (1831-1889), originaire d'Arménie. Les orientalistes Ariel Pavet de Courteille (18211889) et Casimir Barbier de Meynard (1827-1908) publient, le premier un Dictionnaire de turc oriental, le second un Dictionnaire turc-français (1885-1887). Le linguiste polonais Joseph Kowalewski (1800-1878) fait paraître un Dictionnaire mongol-russe-français (1844-1846). Un Dictionnaire kurde-français est à mettre à l'actif de l'orientaliste allemand Ferdinand Justi (1837-1902). Plus exotiques encore, le Dictionnaire étymologique chinoisannamite-latin-français (1867 ff.) de Jean-Pierre Pauthier (1801-1873), ainsi que les Dictionnaires javanais-français (1870), malais-français (1875) et français-malais (1880) de Pierre Favre (1812-1887).

Par une démarche inverse se manifeste en France le souci d'étendre la lexicographie aux facettes dialectales, voire sociales de la langue. Cette préoccupation se concrétise dans des ouvrages comme le Dictionnaire celto-breton ou breton-français (1821) et le Dictionnaire français-breton (1847) de Jean Le Gonidec (1775-1838) ou le Dictionnaire bretonfrançais (1895) de Pierre de Châlons, comme le Dictionnaire provençal-français (1846) et le Vocabulaire français-provençal (1849) de Simon Honnorat (1783-1852) ou le Trésor du félibrige (1878-1886) provençal-français de Frédéric Mistral (1831-1914), comme le Dictionnaire thématique français-argot (1897) de Jules Lermine (1839-1915).

Les lexicographes britanniques sont partagés semblablement entre l'inventaire du passé et du local - comme Thomas Wright dans ses Anglo-Saxon and Old English Vocabularies (1884) ou Charles Mackay (1814-1889) dans son Dictionary of Lowland Scotch (1888) - et l'exploration de territoires nouveaux. Ainsi, dès 1810, l'orientaliste William Carey (1761-1834) publie un Marathi Dictionary, suivi d'un Bengali Dictionary en 1818; la langue mahrate sera encore traitée par J. Molesworth dans son Dictionary of Marahti and English (1857). Robert Morrison (1782-1834) est l'auteur du premier Chinese-English Dictionary (1815-1823); Walter Medhurst (1796-1857), celui du premier English-Japanese Dictionary (1830). Le diplomate James Redhouse (1811-1892) compose un English-Turkish and Turkish-English Dictionary (1856), tandis qu'un vaste ArabicEnglish Lexicon (1863-1892) et un Abridged English-Persian Dictionary (1883) paraissent sous la plume respective des orientalistes Edward Lane (1801-1876) et Edward Palmer (1840-1882), et que Richard Cleasby publie le premier Icelandic-English Dictionary (1874). Les dialectes indiens ou malais n'échappent pas aux investigations des lexicographes, comme le prouvent le Dictionary of the Otchipwe language explained in English 
(1853) de F. Baraga, le Dictionary of the language of the Micmac Indians (1888) de S. Rand ou le Dictionary of the Central Nicobarese Language (1888) d'E. Man.

L'allemand également, en dehors des combinaisons traditionnelles avec les principales langues de culture, se voit associé à quantité de langues nouvelles, tant européennes que plus lointaines. Le professeur tchèque Joseph Jungmann (1773-1847) publie un Tschechisch-deutsches Wörterbuch (1835-39); son collègue, Anton Ianejitch (1828-1869), fait de même pour le slovène. Pour le hongrois paraissent le Ungarisch-deutsches Wörterbuch (1836), de Janos Fogarasi (1801-1878), et divers dictionnaires compilés par le philologue Maurice Ballagi (1815-1891). L'orientaliste hongrois Armin Vambery (18321913), qui découvrit la parenté entre le turc et le magyar, donne un Deutsch-türkisches Wörterbuch (1858). Plusieurs langues asiatiques sont abordées pour la première fois: un Mongolisch-russisch-deutsches Wörterbuch (1835) et un Tibetisch-deutsches Wörterbuch (1841) paraissent sous la plume de l'orientaliste Isaac Schmidt (1779-1847), tandis que le linguiste Hans Conon von der Gagelentz (1807-1874), qui étudia aussi les dialectes des Indiens d'Amérique, publie un Mandschurisch-deutsches Wörterbuch (1864). L'orientaliste Adolf Wahrmund (1827-1913), quant à lui, se signale par un Arabisch-deutsches und deutsch-arabisches Handwörterbuch (1874-1877), tandis que J. Krönlein s'intéresse à la langue des Hottentots dans son Wortschatz der Khoi-Khoin (1889).

Cette floraison lexicographique, illustrée ici par des exemples tirés des domaines français, anglais et allemand, ne se limite évidemment pas à ces seules langues, et quelques ouvrages au hasard suffisent à s'en convaincre: en Lituanie, le littérateur Simon Daukantas (1793-1864) compose un Dictionnaire polono-lituanien; en Turquie, l'écrivain et homme politique Ahmed Wefik (1819-1891) publie un Dictionnaire turc-osmanli; en Finlande, Elias Lönnrot (1802-1884), l'artisan de la renaissance littéraire finnoise, se distingue en outre par un Dictionnaire finnois-suédois (1866-1880), etc.

\section{LE XX SIÈCLE}

Au XX' $\mathrm{X}^{\mathrm{e}}$ siècle, l'internationalisation des échanges s'accompagne d'une activité lexicographique dont l'ampleur ira sans cesse grandissant.

\section{Avant 1914-1918}

Dans les années qui précèdent la Première Guerre mondiale, elle est encore modeste, même si elle donne naissance à des ouvrages comme 1'Italian and English Dictionary (1901) de H. Edgren, comme le Cassell's French-English, English-French Dictionary (1904) d'A. de Curzon, comme l'Appleton's English-Spanish and Spanish-English Dictionary (1903) d'Antonio Cuyas, comme l'Illustrated Practical English-Armenian Dictionary (1910) de Z. Papazian, comme le Russisch-deutsches Wörterbuch (1911) d'I. Pawlowski, comme, surtout, le Langenscheidt's Encyclopaedic Dictionary of the English and German Languages (1908) d'Eduard Muret et Daniel Sanders, encore actuellement le plus complet des dictionnaires allemand-anglais, issu d'un projet qui remonte à 1869 .

\section{Après 1914-1918}

La guerre finie, le français, qui avait jusque-là dominé les relations diplomatiques en Occident, doit partager son monopole avec l'anglais, devenu son égal. Pour les lexicographes, c'est le signal de la mise en chantier d'un grand dictionnaire français-anglais, qui se concrétise dans le Harrap's Standard French and English Dictionary (1934-1939) de J. Mansion, incontestablement le meilleur pour cette combinaison de langues, bien qu'il faille mentionner aussi l'Oxford Practical French-English Dictionary for Englishspeaking Countries (1940) de G. Goodridge. L'entre-deux-guerres s'enrichit aussi d'un 
Gran diccionario inglés-español, español-inglés (1928), des frères Arturo et Antonio Cuyas, qui n'a rien de commun avec l'ouvrage publié par Appleton, d'un Dizionario italianoinglese, inglese-italiano (1930), de N. Spinelli, d'un English-Russian and Russian-English Dictionary (1931-1935), de V. Myuller et S. Boyanus, d'un Diccionario inglês-português, portugês-inglês (1933), de J. Albino Ferreira, d'un Ossetisch-russisch-deutsches Wörterbuch (1934), de V. Myuller, d'un New English-Russian Dictionary, de M. Golovinski, d'un English-Lituanian Dictionary (1939), de H. Pewtress et T. Gerikas, pour ne citer que quelques ouvrages parmi beaucoup d'autres.

\section{Après la Seconde Guerre mondiale}

C'est toutefois la Seconde Guerre mondiale, avec les bouleversements politiques profonds qu'elle déclenche, qui va provoquer un nouvel essor de la lexicographie bilingue et l'orienter vers des combinaisons jusque-là impratiquées. La satellisation d'une partie de l'Europe par l'Union Soviétique confère aux langues de ces pays une importance nouvelle, en particulier pour une Allemagne de l'Est entrainée dans la même orbite. Tout comme la colonisation avait commandé la connaissance d'un certain nombre de langues exotiques, l'intérêt que l'impérialisme marxiste porte aux pays en voie de développement va se traduire par des dictionnaires consacrés aux langues de leurs habitants. Un État multilingue comme l'URSS se trouve par ailleurs confronté, à l'intérieur même de ses frontières, à la barrière de la communication entre ses diverses communautés: Dans plusieurs pays européens, la présence de travailleurs immigrés oblige à se pencher sur le problème de leur compréhension. Dans d'autres, les régionalismes politiques poussent à une résurgence des dialectes. Tous ces facteurs, joints à l'omniprésente internationalisation des relations diplomatiques, commerciales, scientifiques, culturelles, font que l'on assiste à un véritable éclatement des besoins lexicographiques dans toutes les directions. Les deux Allemagne, la fédérale et la démocratique, en fournissent un bel exemple.

En Allemagne de l'Ouest, des maisons comme Langenscheidt, Brockhaus, Duden, Brandstetter, Bertelsmann et d'autres ont bien sûr toutes leurs dictionnaires pour les langues de grand rayonnement. Les deux premières se sont spécialisées dans les ouvrages illustrés, Brockhaus avec ses Bildwörterbuch französisch-deutsch, deutsch-französisch d'E. Pfohl (30 édition en 1956, par P. Benoit), Illustrated Dictionary German-English, English-German (1961), de H. Motekat et J. Bourke, Novo Michaelis Dicionario ilustrado (1958-1961), de F. Pietschke pour le portugais, Bildwörterbuch deutsch-italienisch (1969), d'U. Kemper, et l'homologue italien-allemand (1974) d'A. Cozzi, etc.; Duden avec un Duden français. Dictionnaire en images (1936), un Duden Pictorial Encyclopaedia in 5 Languages ( $2^{\mathrm{e}}$ éd., 1958) et un English Duden ( $2^{\mathrm{e}}$ éd., 1960), un Duden español. Diccionario por la imagen ( $2^{\mathrm{e}}$ éd., 1963), un Duden italiano. Dizionario figurato ( $2^{\mathrm{e}}$ éd., 1964), même un Svenska Duden-Bildlexikon (1966), etc. Dans la catégorie des dictionnaires traditionnels, Brandstetter a Bertaux-Lepointe pour le français, Wildhagen-Héraucourt pour l'anglais, Slaby-Grossmann pour l'espagnol, etc.; Langenscheidt, qui réserve à ces langues majeures ses grands dictionnaires encyclopédiques, comme le Muret-Sanders pour l'anglais (réédition 1962-1963 d'O. Springer), comme le Sachs-Villatte pour le français (dernière édition en 1979, par E. Weis, qui avait déjà signé, avec H. Mattutat, un Wörterbuch der deutschen und französischen Sprache en 1959), comme le Grosswörterbuch italienisch (1964), de V. Macchi pour l'italien, leur consacre aussi des ouvrages d'une envergure plus modeste, quoique de qualité comparable, tels le Handwörterbuch deutsch-englisch (1959), de H. Messinger, et son homologue anglais-allemand (1964), le Handwörterbuch deutsch-französisch (1961), d'E. Lange-Kowal, et son homologue françaisallemand (1976), le Handwörterbuch italienisch-deutsch, deutsch-italienisch (1965), de 
P. Giovanelli et H. Frenzel, le Handwörterbuch spanisch-deutsch, deutsch-spanisch (1971), de H. Müller, G. Haensch et E. Prada, etc.

La même collection s'ouvre aux langues de l'Europe de l'Est avec un Handwörterbuch polnisch-deutsch (1957), de P. Kalina, et son homologue allemand-polonais (1961), de J. Chodera et S. Kubica, un Handwörterbuch tschechisch-deutsch, deutsch-tschechisch (1963), de J. Volny, un Handwörterbuch ungarisch-deutsch, deutsch-ungarisch (1965), d'E. Halasz, etc.

Mais Langenscheidt n'est pas le seul éditeur à se laisser guider par les circonstances politiques ou sociales. D'autres que lui produisent des dictionnaires dictés soit par la présence de main-d'œuvre immigrée, soit par l'expansion industrielle allemande aux quatre coins du monde. À la première catégorie appartiennent un Türkisch-deutsches Wörterbuch (1972), de K. Steuerwald, ou un Dicionário idiomático português-alemáo (1979), de H. et L. Schemann; dans la seconde peuvent se ranger le Deutsch-persisches Wörterbuch (1963), de W. Eilers, et le Persisch-deutsches Wörterbuch (1971), de F. Sobhani, le Deutscharabisches Wörterbuch (1968), de G. Schregle, le Deutsch-chinesisches Wörterbuch (1970), de H. Wilhelm, le Wörterbuch der koreanisch-deutschen Sprache (1971), d'A. Eckardt, le Deutsch-japanisches Wörterbuch (1977), d'E. Breitung.

L'Allemagne de l'Est offre un tableau assez semblable, avec parfois des traits plus accusés ou d'inspiration différente. Si elle ignore le problème des travailleurs immigrés, par contre, son appartenance au bloc soviétique la contraint à s'intéresser davantage aux langues de ses alliés, à commencer par le russe. En 1959 paraît un Bildwörterbuch deutsch und russisch, suivi d'un Deutsch-russisches Wörterbuch (1962), par E. Daum et W. Schenck, d'un Taschenwörterbuch deutsch-russisch (1964), par R. Ruzicka, d'un Russisch-deutsches Wörterbuch, par H. Bielfeldt, qui connaît déjà sa quatorzième édition en 1968, etc. Même la langue de l'une des républiques soviétiques se voit associée à l'allemand dans le Georgisch-deutsches Wörterbuch (1971), de K. Tschenkeli et Y. Marchev.

Il en va de même pour la plupart des pays satellites: Taschenwörterbuch polnischdeutsch (4 ${ }^{\mathrm{e}}$ éd., 1968), de L. Jakowczyk et W. Reinholz, Taschenwörterbuch tschechischdeutsch (4éd., 1968), de R. Fischer, Taschenwörterbuch deutsch-tschechisch (4e éd., 1968), de F. Widimsky et B. Mencak, Taschenwörterbuch bulgarisch-deutsch (1963), de P. Rankov, Taschenwörterbuch deutsch-slowakisch ( $2^{\mathrm{e}}$ éd., 1968), de V. Blanar, Bildwörterbuch deutsch und rumänisch (2e éd., 1969), d'E. Silzer, Wörterbuch albanisch-deutsch (1977), d'O. Buchholz, etc. Une liste établie par H.-J. Bäse (Lebende Sprachen 1966-1967) ne relève pas moins d'une quarantaine de dictionnaires généraux associant l'allemand au russe, au polonais, au tchèque, au sorabe, au serbo-croate, au slovène, au bulgare, au roumain, au hongrois, au macédonien, à l'ukrainien.

Tout à l'opposé, l'intérêt idéologique ou technologique que la RDA porte au tiers monde engendre des dictionnaires comme le Persisch-deutsches Wörterbuch (2e éd., 1968), de H. Junker et A. Bozorg, le Swaheli-deutsches Wörterbuch (1963), de H. Hoffmann, le Deutsch-arabisches Wörterbuch (1967), de G. Kahlo et R. Bärwinkel, le Hindi-deutsches Wörterbuch (1971), d'E. Klemm, le Chinesisch-deutsches Wörterbuch (2e éd., 1972), de M. Piasek, le Japanisches-deutsches Zeichenlexikon (1977), de W. Wernecke, etc.

En Grande-Bretagne et aux États-Unis, autres grands de la lexicographie, les principales maisons spécialisées - Oxford, Cassell, Dent, Cambridge, Harrap, Holt, Appleton, etc. - possèdent évidemment aussi des dictionnaires pour la plupart des langues usuelles. Qu'il suffise de mentionner, pour le français, The Concise Oxford French Dictionary (1958), de A. et M. Chevalley, et Everyman's French-English and English-French Dictionary (1962), de J.-P. Vinay et al., qui offre la particularité d'être le premier à faire référence à l'usage canadien. Pour l'allemand, on peut citer le Cassell's German and English Dictionary (1957), de H. Betteridge, et l'excellent Harrap's Standard German and English 
Dictionary (1963), de T. Jones. Le néerlandais est traité par F. Prick van Wely dans le Cassell's Englisch-Dutch and Dutch-English Dictionary. Pour l'espagnol, à côté de l'excellent Holt Spanish and English Dictionary (1955), d'E. Williams, il y a aussi le Cassell's Spanish Dictionary (1959), d'E. Peers et al. Le portugais a le Novo dicionário da lingua portuguesa e inglesa (1945), de H. Michaelis, et The New Appleton Dictionary of the English and Portuguese Languages (1964), d'A. Houaiss et C. Avery. Pour l'italien, le grand Italian Dictionary, d'A. Hoare, publié au lendemain de la Première Guerre mondiale et épuisé, devient The Cambridge Italian Dictionary (1962), dans l'édition actualisée de B. Reynolds.

Les dictionnaires consacrés aux langues nordiques, s'ils ne sont pas nécessairement l'œuvre de lexicographes anglo-saxons, se basent le plus souvent sur l'anglais. Une bibliographie dressée par I. Gullberg (Lebende Sprachen 1981) énumère 35 dictionnaires pour le suédois, 19 pour le danois, 19 pour le norvégien et 5 pour l'islandais, tous en combinaison avec l'anglais ou l'allemand. Pour des raisons de regroupement logique, on peut donc parler ici du Svensk-engelsk ordbok (1944), de W. Harlock et al., qui forme avec son homologue Engelsk-svensk ordbok (1955), de K. Kärre et al., la meilleure paire pour le suédois; du Dansk-engelsk ordbog (1954-1956), de H. Vinterberg et C. Bodelsen, qui constitue, avec l'Engelsk-dansk ordbog (1964) de B. Kjaerulff Nielsen, la meilleure paire pour le danois; du Norwegian-English Dictionary (1965), d'E. Haugen et al., qui forme avec l'English-Norwegian Dictionary $\left(1911 ; 2^{\mathrm{e}}\right.$ éd., 1948), de T. Gledfisch et al., le couple le plus complet pour le norvégien; de l'Icelandic-English Dictionary ( $2^{\mathrm{e}}$ éd., 1957), de R. Cleasby et G. Vigfusson, qui constitue, avec l'English-Icelandic Dictionary (1952) de S. Bogason, la meilleure paire pour l'islandais. On peut y ajouter, pour le finnois, le FinnishEnglish Dictionary (1919; $2^{\mathrm{e}}$ éd., 1956), de V. Alanne, et l'English-Finnish Dictionary (1939; 4e éd., 1964), d'A. Tuomikoski et A. Slöör.

Les langues slaves sont représentées par le Russian-English and English-Russian Dictionary (1953), de L. Segal, et un dictionnaire du même nom (1973), de R. et S. Fleming, ainsi que par un English-Czech and Czech-English Dictionary (1959), de J. Prochazka. Pour le turc paraissent $A$ Turkish-English Dictionary (1945), d'A. Vahit et, en deux volumes, le Turkish-English and English-Turkish Dictionary (1952), de H. Hony et Fahir Iz.

Parmi les langues plus exotiques, il faut citer le Chinese-English Dictionary (1947), de R. Matthews, le plus complet qui soit, qui forme, avec le New English-Chinese Dictionary (1950) d'Y. Cheng et C. Tsao, la meilleure paire disponible; le Twentieth Century English-Hindi Dictionary (1951), de S. Bhandari; A Dictionary of Modern Written Arabic (1976), de J. Milton Cowan, pour le sens anglais-arabe; et jusqu'à un English-Hawaian Dictionary (1967), de H. Hitchcock, un Dictionary of the Maori Language (1971), de H. Williams, un Luganda-English Dictionary (1972), de J. Murphy, un English-Amharic Dictionary (1973), de W. Leslau, ou un English-Kikuyu Dictionary (1975), d'A. Barlow.

Par ailleurs, les aspects argotique, et familier, de la langue anglaise ne sont pas négligés par les lexicographes, tant américains que britanniques, si l'on en juge par le Dictionary of French and American Slang (1965) anglais-français et français-anglais, de M. Leitner et J. Lanen, ou le Harrap's French-English Dictionary of Slang and Colloquialisms (1970, uniquement français-anglais), de J. Marks.

En France, comme ailleurs, le marché des dictionnaires bilingues est entre les mains d'un petit nombre d'éditeurs spécialisés - Larousse, Hachette, Hatier, Garnier, etc. qui couvrent toutes les grandes langues occidentales. Pour ne prendre que l'exemple de l'anglais, il y a le Dictionnaire français-anglais et anglais-français (1956), d'E. Clifton et J. McLaughlin, chez Garnier, le Dictionnaire moderne français-anglais et anglaisfrançais (1960), de M. Dubois, chez Larousse, le Dictionnaire français-anglais (1961), de Ch. Cestoe, chez Hatier, etc., auxquels il convient d'ajouter le premier dictionnaire 
bilingue produit par la Société du Nouveau Littré (SNL-Le Robert), le Dictionnaire Robert-Collins français-anglais et anglais-français (1978), de S. Atkins et P. Cousin. Pour l'usage américain, E. Deak publie un premier Dictionnaire d'américanismes (1956) dont la deuxième édition, en 1957, passe de 232 à 700 pages.

S'il faut illustrer l'éclectisme de la lexicographie française, il suffit de citer des ouvrages comme le Grand dictionnaire hongrois-français (1958), d'A. Eckardt, d'une part, comme les Dictionnaires swahili-français, de Ch. Sacleux (1939) ou d'A. Lenselaer (1975), et d'autre part, le Dictionnaire moderne français-tahitien et tahitien-français (1973), d'Y. Lemaître.

Enfin, un Petit dictionnaire provençal-français (1961), d'E. Lévy, ou un Dictionnaire français-argot (1968), de J. Marcillac, montrent que les aspects dialectaux et sociaux de la langue gardent toute l'attention des lexicographes.

Dans les autres pays latins - Italie, Espagne, Portugal —, l'activité lexicographique n'est pas en reste, surtout dans les deux premiers. En Italie, le Nuovo dizionario moderno delle lingue italiana e inglese (2 vol., 1950-1958), d'A. Severino et L. Gualtieri, fait autorité pour la langue anglaise, laquelle est traitée aussi dans des ouvrages moins volumineux comme le Dizionario inglese-italiano, italiano-inglese (1961), de M. Hazon, et le Dizionario italiano-inglese e inglese-italiano (1961), de G. Orlandi. Pour l'allemand paraissent le Dizionario italiano-tedesco, tedesco-italiano (1958-1959), d'E. Bidoli et G. Cosciani, ainsi que le Vocabolario tedesco-italiano, italiano-tedesco (1971), d'A. Deidda. Parmi les ouvrages réservés au français, l'un des plus récents est le Dizionario italiano-francese e francese-italiano (1971), de G. Sbrulli et T. Biffoli.

En Espagne, la collaboration entre la maison d'édition Sopeña et le lexicographe E. Martínez Amador débouche sur quelques ouvrages de qualité, à commencer par le Standard Spanish-English Dictionary and Diccionario inglès-español $(1946 ; 4 \mathrm{e}$ éd., 1958), qui reste le meilleur, malgré le Modern Spanish Dictionary (1953), de M. Raventós, déjà excellent, le New Revised Velásquez Spanish and English Dictionary (1960), réédité par I. Navarro Hinojosa, ou le Nuevo diccionario general inglés-español. New Comprehensive Spanish-English Dictionary (1966), d'U. di Benedetto et al.; Martínez Amador est aussi l'auteur d'un Diccionario español-francés y francés-español $(1957 ; 2 \mathrm{e}$ éd., 1961) et d'un Diccionario italiano-español y español-italiano (1957). Quant à la maison Sopeña, elle publie encore le Diccionario alemán-español y español-alemán (1957), de F. Müller. En 1960, J. Pensado et E. Ruiz font paraître un Diccionario portugués-español y español-portugués; en 1965, Laszlo et Galdi font de même pour le hongrois, avec leur Diccionario español-hungaro. En Espagne comme en France, l'intérêt que les lexicographes portent aux langues régionales par rapport à la langue nationale donne naissance à des ouvrages comme le Diccionario castellano-vasco (1958), de P. Beralopez Mendizabal, pour le basque, le Diccionario enciclopédictionnaire galleco-castellano (1962), d'E. Rodriguez Gonzalez, ou le Diccionario galego-castelán e Vocabolario castelángalego (1968), de L. Carré Alvarellos, pour le galicien.

Le Portugal, malgré une activité lexicographique toujours moins intense qu'ailleurs, s'enrichit néanmoins d'un Dicionario moderno português-inglês (1954), de M. Teixeira de Oliveira, l'un des plus complets pour le sens portugais-anglais, et même d'un Dicionario português-ruso (1960), de S. Brandon.

En URSS, l'éventail des dictionnaires bilingues embrasse aussi bien les langues européennes que les autres. Pour les premières, on peut signaler le bon Russian-English Dictionary (1965), d'A. Smirnitski, le Dictionnaire russe-français (9e éd., 1969), de L. Tcherba et M. Matucevitch, et son homologue français-russe (6e éd., 1971), de K. Ganchina, ou le Dictionnaire pratique russe-français (4' éd., 1978), d'A. Zalizniak, et son homologue français-russe ( $2^{\mathrm{e}}$ éd., 1978), de N. Kobrina et al., le Diccionario ruso-español ( $2^{\mathrm{e}}$ 
éd., 1963), de Y. Yaselman, etc. Pour les autrés, il y a notamment le Dictionnaire russechinois (1952), d'l. Ochanin, et son homologue chinois-russe (3e éd., 1953), de Ch. Chen, le Dictionnaire hindi-russe ( $2^{\mathrm{e}}$ éd., 1959), de W. Beskrowny, le Dictionnaire vietnamienrusse (1960), d'I. Glebov, le Dictionnaire indonésien-russe (1960), de R. Korigodski, et bien d'autres. Par ailleurs, la diversité des communications entre les diverses républiques est illustrée par des ouvrages comme le Dictionnaire russe-biélorusse (1965), de S. Grabtchikov, le Dictionnaire de la langue tchouvache (1973), d'A. Gorchkov, le Dictionnaire de la langue kazake (1974), d'A. Ibatov, le Dictionnaire de la langue yakoute (1974), de N. Grigorev, etc.

Aux Pays-Bas, des éditeurs comme Wolters, Kramers, Van Goor, Prisma et autres Standaard jouent le même rôle que leurs confrères français, anglais ou allemands : leurs dictionnaires bilingues couvrent la plupart des langues de grande diffusion. Pour l'anglais, l'ouvrage le plus complet est l'Engels woordenboek anglais-néerlandais et néerlandaisanglais, de R. ten Bruggencate et A. Broers, qui atteint déjà sa seizième édition en 1963, par les soins de R. Zandvoort et J. Gerritsen. Pour le sens néerlandais-anglais seul, il est concurrencé par le Groot Nederlands-Engels Woordenboek (1950), de H. Jansonius. De même, pour l'allemand, il y a Kramers Handwörterbuch Niederländisch allemandnéerlandais et vice-versa, de J. van Dam, et, pour le seul sens allemand-néerlandais, Wolters Duits Woordenboek, d'I. van Gelderen, réédité en 1971, par W. Wallis. Pour le français, à côté d'un Woordenboek Nederlands-Frans en Frans-Nederlands, de Kramers, ou du grand Dictionnaire français-néerlandais et néerlandais-français, de J. Grootaers, il convient surtout de distinguer les deux volumes du Fransch Woordenboek (1904-1907), de K. Gallas, devenu après la guerre 1939-1945 le Nieuw Frans-Nederlands en NederlandsFrans Woordenboek (1954-55), incontestablement le plus complet et le plus fiable pour cette combinaison, comme le Spaans-Nederlands Woordenboek (2 vol., 1963), de S. Voster, l'est pour celle de l'espagnol et du néerlandais.

L'abondance des exemples cités éclaire à suffisance l'étendue des horizons explorés par la lexicographie bilingue au XXe siècle, sans pour autant en épuiser toutes les richesses. Il existe encore des dictionnaires qui combinent le français, le néerlandais, l'anglais ou l'allemand avec le danois, le norvégien, le suédois, le russe, le polonais, le portugais, le turc et l'arabe; qui combinent le français, le néerlandais ou l'anglais avec le chinois; qui combinent le français, le néerlandais ou l'allemand avec le tchèque, le cerbo-croate et le hongrois; qui combinent le français ou l'anglais avec le japonais, le laotien, le sanskrit, l'indonésien et le swahili; qui combinent le néerlandais ou l'allemand avec l'islandais et le roumain; qui combinent le français ou l'allemand avec le bulgare, le finnois et le slovaque; qui combinent le français avec l'albanais, l'arménien, le basque, le cambodgien, le corse, le dogon, le fang, l'hébreu, le lingala, le munnkutuba, le somalien le tahitien, le thai et le vietnamien; qui combinent l'anglais avec l'afrikaans, le bengali, le coréen, l'esquimau, le gallois, le gujarati, le penjabi, le tagalog, le tibétain, l'ukrainien, etc.

\section{Le traducteur et le dictionnaire}

Ces auxiliaires indispensables à la communication entre les occupants d'un monde qui va sans cesse se rétrécissant, peut-on encore leur appliquer les paroles un rien méprisantes de Valéry Larbaud, qui ne voulait voir dans les dictionnaires bilingues «que des esclaves, ou mieux des affranchis faisant fonctions d'huissiers et d'interprètes» -, même s'il admettait plus tard avoir été injuste et ingrat envers eux en soulignant que «nous-mêmes, traducteurs d'aujourd'hui, pourrions et devrions contribuer à la préparation des futurs grands bilingues». 